\title{
ON DEFINABLE COMPLETENESS FOR ORDERED FIELDS
}

\begin{abstract}
A b s t r a c t. We show that there are 0-definably complete ordered fields which are not real closed. Therefore, the theory of definably with parameters complete ordered fields does not follow from the theory of 0-definably complete ordered fields. The mentioned completeness notions for ordered fields are the definable versions of completeness in the sense of Dedekind cuts. In earlier joint work, we had shown that it would become successively weakened if we just required nonexistence of definable regular gaps and then disallowing parameters. The result in this note shows reducing in the opposite order, at least one side is sharp.
\end{abstract}

Received 27 October 2018

Keywords and phrases: ordered field; 0-definably complete; real closed field. AMS subject classification: Primary 03C64; Secondary 12L12. 


\section{Introduction}

Real closed fields are precisely the definably with parameters Dedekind complete ordered fields. We show that a non-real-closed field may still be 0 -definably complete. This answers a natural question, as in [3, Problem 17(i)], we raised earlier with J. S. Eivazloo. In the main step, for a certain ordered field of generalized power series, it is shown that the set of infinitesimals is not 0-definable (the latter is due to Lou van den Dries).

In $[5,4]$ we had already shown the non-implication for the regular cut variant. Recall that for an ordered field $K$, a gap $C \subset K$ (namely, a cut which has no least upper bound in $K$ ) is regular if for each $\epsilon \in K^{>0}$ we have that $C+\epsilon \nsubseteq C$ (i.e., it is of zero distance to its complement). The ordered field $K$ is called Scott complete if it does not have any proper extensions to an ordered field in which it is dense, equivalently it does not have any regular gaps.

Among the notions of definably (with or without parameters) Scott complete ordered fields we considered in [5] were $D_{p} S_{r c} C O F$ in the parameter case and $D_{\emptyset} S_{r c} C O F$ in the parameter-free case. Their models are ordered fields with no definable regular gaps with or without parameters respectively. We showed there that if an ordered field $K$ is a proper dense sub-field of its real closure, then $K \not \models D_{p} S_{r c} C O F$ (see [5, Lemma 3.1]).

As we mentioned already, definably complete (with parameters) ordered fields are exactly the real closed fields, in notation: $R C F \equiv D_{p} D C O F$. A weaker form of half of this fact is $R C F \vdash D_{p} S_{r c} C O F$. However the converse of the latter fails as we record below.

Fact 1.1. We have $D_{p} S_{r c} C O F \nvdash D_{p} D C O F$ (that is $D_{p} S_{r c} C O F \nvdash$ $R C F)$.

Indeed there are Scott complete ordered fields which are not real closed. One may take the Scott completion of any ordered field which is not dense in its real closure, e.g. $\mathbb{R}(x)$ with $x$ infinitely large (no rational function of $x$ is between $\sqrt{x}$ and $2 \sqrt{x}$ for example).

The following was mentioned in [5, Note added in proof], and in [4, Below Question 5.1].

Fact 1.2. We have $D_{\emptyset} S_{r c} C O F \nvdash D_{p} S_{r c} C O F$.

Now consider the relaxing of $D_{p} D C O F$ again in two steps but in the opposite order, first dropping parameters and then restricting to regular 
gaps. In the next section, we show that there are 0-definably complete ordered fields which are not real closed, i.e. $D_{\emptyset} D C O F \nvdash D_{p} D C O F$ (see Theorem 2.1). The status of the other half of this reverse weakening is left as a question:

Question 1.1. Is it the case that $D_{\emptyset} S_{r c} C O F \nvdash D_{\emptyset} D C O F$ ?

It is known that the field of rational functions $\mathbb{R}(x)$, ordered with $x>\mathbb{R}$, 0 -defines the irregular gap of infinitesimals. One may be able to argue that this field does not 0-define any regular gaps.

\section{0-definably complete ordered fields need not be real closed}

Recall that for an ordered field $F$ and ordered abelian group $G$, the generalized power series field $F\left(\left(t^{G}\right)\right)$ consists of functions $G \rightarrow F$ with well ordered support (making $t$ a positive $F$-infinitesimal) with the obvious addition and multiplication.

Another ingredient we need is the following. Robinson-Zakon, see [6] (and [7] for some of the proofs), defined an ordered abelian group $M \neq\{0\}$ to be regularly dense if for any positive integer $n$ and elements $a, b \in M$ with $a<b$, there is an element $u \in M$ such that $a<n u<b$. Every dense additive subgroup of $\mathbb{R}$ is regularly dense. In particular, the ordered (additive) group of dyadic rationals (the standard rationals which have a representation where the denominator is a power of 2) is regularly dense. This group is going to appear in the proof of our theorem below.

We need the Robinson-Zakon [op. cit.] necessary and sufficient condition for the elementary equivalence of two regularly dense groups $A$ and $B$, namely they have the same prime invariants for every prime $p$. This means the quotient groups $\frac{A}{p A}$ and $\frac{B}{p B}$ are either both infinite (not necessarily of the same cardinalities), or they are both of the same finite order.

We also employ part of the Ax-Kochen-Ershov Principle [1, 2]: Two valued fields both of which with residue fields of characteristic 0 , are elementarily equivalent if and only if their residue fields are elementarily equivalent (in the language of ordered rings) and their value groups are elementarily equivalent (in the language of ordered groups). 
Lemma 2.1. (L. van den Dries) The set of infinitesimals of $\mathbb{R}\left(\left(t^{\Gamma}\right)\right)$, where $\Gamma$ is the ordered (additive) group of dyadic rationals, is not 0-definable.

Proof. (This lemma and its proof are due to L. van den Dries, private communication.) With $\Gamma$ as in the lemma, let $\Gamma^{\prime}=\mathbb{Q} \oplus \Gamma$ be ordered so that the summand $\mathbb{Q}$ is convex in $\Gamma^{\prime}$.

We claim that $\Gamma \equiv \Gamma^{\prime}$. To see this, note that $\Gamma^{\prime}$ is dense since both summands $\mathbb{Q}$ and $\Gamma$ are dense. Also, the first direct summand $\mathbb{Q}$ is divisible, therefore the prime invariants of $\Gamma^{\prime}$ are the same as those of $\Gamma$ (that is $p$ itself, for any $p$, not any higher power of $p$ ). Therefore, by the RobinsonZakon criterion cited above, $\Gamma \equiv \Gamma^{\prime}$.

Let $F=\mathbb{R}\left(\left(t^{\Gamma}\right)\right)$ and $K=\mathbb{R}\left(\left(t^{\Gamma^{\prime}}\right)\right)$. By the Ax-Kochen-Ershov principle mentioned above, we have $F \equiv K$ as they even have the same residue field $\mathbb{R}$ of characteristic zero, and we have shown the value groups $\Gamma$ and $\Gamma^{\prime}$ are elementarily equivalent.

Now the natural valuation $v: K^{\neq 0} \rightarrow \Gamma^{\prime}$ can be coarsened to a valuation $v^{*}: K^{\neq 0} \rightarrow \Gamma^{\prime} / \mathbb{Q}=\Gamma$ and by the AKE principle again, $(K, v) \equiv\left(K, v^{*}\right)$ as valued fields. Now these two valued fields have the same underlying field $K$ but different sets of infinitesimals. E.g., $u=t^{(1,0)}$ as an element of $(K, v)$ has the positive value $(1,0)$, so $u$ is an infinitesimal in $(K, v)$. But as an element of $\left(K, v^{*}\right)$, it has value 0 and is therefore not infinitesimal. Hence the infinitesimals of $K$ do not form a 0 -definable set and consequently the same is true for $F$.

Theorem 2.1. Parameter-free definable Dedekind completeness for ordered fields is strictly weaker than definable completeness:

$$
D_{\emptyset} D C O F \nvdash D_{p} D C O F(\equiv R C F) .
$$

Proof. We use the same field $F=\mathbb{R}\left(\left(t^{\Gamma}\right)\right)$ as in the lemma above. Note that the value group $\Gamma$ of $F$ is not divisible, so our ordered field $F$ is not real closed, equivalently it fails (parameter) definable completeness. An explicit example is the definable (with parameters) gap of all elements which are below $t^{\frac{1}{3}}$.

To show $F$ does not have any 0-definable gaps, we argue as in $[4$, under Question 5.1]. Let $G$ be a (proper) gap in $F$. Assume $G \cap F^{>0} \neq \emptyset$, otherwise an argument similar to the one below would work.

Case 1: Suppose there is an infinitely large element $a \in F^{>0}$, say $a=$ $\lambda t^{-\gamma}+$ Lower Terms, such that $a \in G$. Note that $\lambda, \gamma>0$. Pick $b \in F \backslash G$, 
say $b=\lambda^{\prime} t^{-\gamma^{\prime}}+$ Lower Terms. Since $G$ is downward closed in $F$, we have $a<b$ (and $\gamma \leq \gamma^{\prime}$ ). Take $\gamma^{\prime \prime} \in \Gamma$ such that $\gamma^{\prime \prime}>\frac{\gamma^{\prime}}{\gamma}$. Consider the field automorphism $f$ on $F$ induced by substituting $t^{\gamma^{\prime \prime}}$ for $t$ (there could be infinitely many occurrences of $t$ ). We have $f(a)>b$. So $G$ is not closed under $f$, and hence $G$ cannot be 0-definable.

Case 2: Suppose $G=F_{\text {Finite }}$ (the set of limited elements in $F$, i.e. those bounded in absolute value by a natural number). Then $G$ cannot be 0 -definable in $F$ since otherwise the set of infinitesimals would be 0 definable: $x>0$ is an infinitesimal iff $\frac{1}{x} \notin G$. This would violate the lemma above.

Case 3: Suppose $G$ is a proper gap in the ordered ring $F_{\text {Finite. In }}$ this case, $G \cap \mathbb{R}$ has a least upper bound $a \in \mathbb{R}$. There are the following sub-cases:

Sub-case 3a1, respectively 3a2: Suppose $G$ is the downward closure, respectively complement of the upward closure, of the whole monad in $F$ of $a$. Note that an element $\varepsilon>0$ is an infinitesimal if and only if $G$ is closed under adding $\varepsilon$. By the lemma, $G$ cannot be 0-definable in $F$.

Sub-case 3b: Suppose $G$ intersects the monad of $a$ in $F$ but does not include all of it. Pick $\lambda, \lambda^{\prime} \in \mathbb{R}$ and $\gamma, \gamma^{\prime} \in \Gamma^{>0}$ such that $x:=a+\lambda t^{\gamma}+$ Lower Terms $<G<y:=a+\lambda^{\prime} t^{\gamma^{\prime}}+$ Lower Terms. Again there would be automorphisms $g$ under which $G$ is not closed ( $g$ can be constructed similar to $f$ in case 1 to move $x$ above $y$ ).

Let us note that in case 2 and sub-cases $3 a 1,3 a 2$, one cannot use the argument of case 1 and sub-case $3 \mathrm{~b}$. Any automorphism of $F$ set-wise preserves the monad of any real number, and consequently also $F_{\text {Finite }}$ and both kinds of gaps mentioned in 3a1, 3a2. On the other hand, for case 1 many gaps are not of the monad kind. E.g., the set of elements in $F$ which are less than $t^{-\frac{1}{3}}$ (and similarly elements below $t^{\frac{1}{3}}$ for case $3 \mathrm{~b}$ ).

\section{Acknowledgements}

The author is grateful to Lou van den Dries for Lemma 2.1 and the proof he gave. Thanks are also due to the referee for comments improving the paper. 
Note Added in Proof. Regarding Question 1.1, Somayyeh Tari has communicated to the author that if we expand the language of ordered rings by a new unary predicate symbol, we have the following: If $R$ is a real closed field and $V$ is a proper convex subring of $R$, then any parameter-free definable gap in $(R, V)$ is irregular. This variant of the question would therefore have witnesses to the unprovability. The proof uses quantifierelimination for $\operatorname{Th}(R, V)$, as established by Cherlin and Dickmann in their 1983 paper in Ann. Pure Appl. Logic 25:3 (1983), 213-231.

\section{References}

[1] J. Ax and S. Kochen, Diophantine Problems over Local Fields I, American Journal of Mathematics 87:3 (1965), 605-630.

[2] J. Ax and S. Kochen, Diophantine Problems over Local Fields III, Decidable Fields, Annals of Mathematics (2) 83:3 (1966), 437-456.

[3] L. Bélair, Z. Chatzidakis, P. D'Aquino, D. Marker, M. Otero, F. Point, and A. Wilkie, Open Problems in Model Theory, In: Same editors, Proceedings of the Euro-Conference on Model Theory and Applications, Ravello, Italy, May 27-June 1, 2002, Quad. Mat. 11, Aracne, Rome, 2002, pp. 459-466.

[4] J.S. Eivazloo and M. Moniri, Expansions of Ordered Fields without Definable Gaps, Mathematical Logic Quarterly 49:1 (2003), 72-82.

[5] M. Moniri and J.S. Eivazloo, Using Nets in Dedekind, Monotone, or Scott Incomplete Ordered Fields and Definability Issues, In: P. Simon, editor, Proceedings of the Ninth Prague Topological Symposium, Prague, August 1925, 2001, Topology Atlas, North Bay, ON, 2002, pp. 195-203. Available at: http://at.yorku.ca/p/p/a/e/00.htm.

[6] A. Robinson and E. Zakon, Elementary Properties of Ordered Abelian Groups, Transactions of the American Mathematical Society 96:2 (1960), 222-236.

[7] E. Zakon, Generalized Archimedean Groups, Transactions of the American Mathematical Society 99:1 (1961), 21-40.

Department of Mathematics and Computer Science

Normandale Community College

9700 France Ave S., Bloomington, MN 55431, USA

mojtaba.moniri@normandale.edu 